\title{
VALORES ORIENTADORES DO COMPORTAMENTO E AÇÃO DOS AGRICULTORES DE BASE ECÓLOGICA PERTENCENTES À ASSOCIAÇÃO DOS PRODUTORES DA REDE AGROECOLÓGICA METROPOLITANA (RAMA), PORTO ALEGRE/RS, BRASIL
}

\author{
Value orientations of ecological farmers of Associação dos Produtores da Rede \\ Agroecológica Metropolitana (RAMA), Porto Alegre/RS, Brazil \\ Valores orientativos para el comportamiento y la acción de los agricultores \\ ecológicos de La Red Agroecológica Metropolitana (RAMA), Porto Alegre/RS, \\ Brasil

Leonardo Bohn*
Daniela Garcez Wives**
* Universidade Federal do Rio Grande do Sul
- leobohn@hotmail.com
* Doutora pela Faculdade de Ciências Econômicas da UFRGS
- garcezd@gmail.com

Recebido em 07/01/2020. Aceito para publicação em 15/04/2020

Versão online publicada em 04/07/2020 (http://seer.ufrgs.br/paraonde)

\section{Resumo:}

Sabe-se que o comportamento dos agricultores é orientado a partir de numerosos valores e objetivos e não somente visando à maximização de ganhos financeiros. O entendimento de suas motivações pode ajudar na percepção de como os mesmos agem e tomam suas decisões acerca da gestão e organização dos sistemas produtivos para além de dimensões técnicoeconômicas, auxiliando assim numa melhor compreensão dos diferentes processos de desenvolvimento observados no mundo rural. Nesse sentido, o objetivo central deste artigo é compreender quais são os regimes orientadores de valores dos agricultores de base ecológica de Porto Alegre e arredores pertencentes à Associação dos Produtores da Rede Agroecológica Metropolitana - RAMA. Para tanto, foram aplicados questionários semiabertos junto a 15 agricultores, utilizando-se de teorias da psicologia comportamental para análise dos resultados. Destaca-se que as motivações dos agricultores entrevistados são orientadas principalmente por regimes de valores intrínsecos e expressivos. Ou seja, os mesmos norteiam suas decisões e comportamento na busca de maior qualidade de vida e satisfação pessoal através da agricultura.

Palavras-chave: Motivações. comportamento. tomada de decisão.

\begin{abstract}
:
It is well known that farmers behavior is guided by mutiple values and goals and not simply aiming at maximizing finantial gains. Understanding their motivations can help in the perception of how they act and make their decisions on management and organization of production systems beyond technical and econonic dimensions, helping, thus, to understand the diferent development processes occuring in the rural world. Therefore, the main objective of this article is to perceive what are the value orientations of ecological farmers from
\end{abstract}


Porto Alegre and surroundings and members of Associação dos Produtores da Rede Agroecológica Metropolitana - RAMA. For that reason, semi-open questionnaires were applied to 15 farmers, using behavioral psychology theories to analyze the results. It is noteworthy that these farmers motivations are guided by intrinsic and expressive value orientations. That is, their choices and behavior are shaped in the pursuit of higher quality of life and personal satisfaction through agriculture.

Key-words: Motivations. behavior. decision making.

\begin{abstract}
Resumen:
Se sabe que el comportamiento de los agricultores se basa en numerosos valores y objetivos y no solo para maximizar las ganancias financieras. Comprender sus motivaciones puede ayudarlos a comprender cómo actúan y como hacen sus decisiones sobre la gestión y organización de los sistemas de producción más allá de las dimensiones técnicas y económicas, lo que ayuda a comprender mejor los diferentes procesos de desarrollo observados en el mundo rural. En este sentido, el objetivo principal de este documento es comprender cuáles son los regímenes orientativos de valor de los agricultores de base ecológica en Porto Alegre y alrededores que pertenecen a la Asociación de Productores de la Red Agroecológica Metropolitana - RAMA. Para este propósito, se aplicaron cuestionarios semiabiertos a 15 agricultores utilizando teorías de psicología conductual para analizar los resultados. Es de destacar que las motivaciones de los agricultores entrevistados se guían principalmente por regímenes de valor intrínseco y expresivo. Es decir, guían sus decisiones y comportamiento en busca de una mayor calidad de vida y satisfacción personal a través de la agricultura.
\end{abstract}

Palabras-clave: Motivaciones. comportamiento. toma de decisiones.

\title{
1. Introdução
}

De acordo com a economia neoclássica, a lógica e o comportamento de agricultores na gestão de suas unidades produtivas (UPs) são orientados predominantemente visando à maximização de ganhos monetários (GASSON, 1973; ROMERO \& REHMAN, 2003). No entanto, a partir dos anos 1970, surge uma ampla bibliografia acerca da importância de se considerar motivações não econômicas nos processos decisórios de agricultores em suas explorações agrícolas.

A partir de tais publicações começa a se compreender que na verdade agricultores possuem inúmeros objetivos, os quais são moldados tanto pelo contexto no qual estão inseridos, como por suas características pessoais e socioeconômicas (GASSON, 1973; KERRIDGE, 1978, ONDERSTEIJN et al., 2003). Dessa maneira, entende-se que as unidades agrícolas se encontram no interior de um ambiente dinâmico e em constante mutação, o qual é configurado por fatores externos (político, ambiental e econômico) e fatores internos (relações de produção, sociais e comportamentais), sendo estas consideradas a projeção dos múltiplos objetivos do agricultor e de sua família (CEZAR et al., 2010; RODRIGUES et al., 2010).

Essa multiplicidade de fatores e objetivos relaciona-se com a grande

ParaOnde!?, Porto Alegre, v.13, n.1, p.152-166, 2020.http://seer.ufrgs.br/paraonde 
diversidade existente nos espaços rurais e nas diferentes dinâmicas regionais e locais. Assim, o entendimento dos valores e motivações dos agricultores pode contribuir na percepção das diferentes formas de gestão e organização das UPs para além de dimensões técnico-econômicas e, por conseguinte, numa melhor compreensão dos processos de desenvolvimento rural (WIVES \& MACHADO, 2014).

A utilização de abordagens comportamentais em estudos agrícolas auxiliam na compreensão dos fatores internos atuantes no comportamento dos agricultores. Tal perspectiva, além de levar em conta aspectos relacionados a propriedade, estrutura socioeconomica, meio natural, entre outros, tem como foco principal as construções psicológicas acionadas pelos mesmos na sua tomada de decisão e gestão da propriedade ou empreendimento rural (BURTON, 2004). Entre os fatores influentes inerentes ao agricultor estão: autonomia, personalidade, habilidade em solucionar problemas, aversão à riscos, quantidade e qualidade de informação, personalidade, etc. (MCGREGOR et al., 1996)

Dentre os estudos que utilizam-se desta abordagem comportamental, destaca-se o trabalho pioneiro de Gasson (1973). Para a autora, objetivos são fins ou estados nos quais o indivíduo deseja alcançar, podendo em alguns casos ser o caminho para propósitos mais amplos. Já, valores são fins em si mesmos, os quais servem como um "padrão", influenciando nos modos de vida e meios de ação. Estes são menos suscetíveis a mudanças ao tempo e circunstâncias, constituindo-se como um produto cultural compartilhado por todos os membros de um determinado sistema social.

Segundo Gasson (1973), valores não ocorrem de forma isolada, sendo os mesmos organizados em regimes de orientação ou sistemas, os quais determinam normas e instituições socialmente aceitas para se conquistar os fins desejados. Nesse sentido, a partir de uma investigação empírica, a autora classificou os agricultores de Cambridge na Inglaterra em quatro regimes de orientação diferentes: (a) expressivo, relacionado à realização e à satisfação pessoal através da prática agrícola; (b) instrumental, preocupado com obtenção de um bom (satisfatório) rendimento e na expansão dos negócios; (c) intrínseco, o qual valoriza a agricultura como uma atividade em si mesma e no desfrute e apreciação do trabalho no campo e, (d) social, associado às relações interpessoais e ao reconhecimento e prestígio social de ser agricultor.

Com base nesta perspectiva são inúmeros os trabalhos encontrados na literatura que analisam as orientações influentes nos agricultores principalmente na tomada de decisão, gestão e organização de suas unidades produtivas no Brasil (CEZAR, 1999; ANDREATTA, 2009; ANDRADE, 2010; WIVES \& MACHADO, 2014; SENGER, 2016; PANNO, 2016) e no mundo (GASSON, 1973; RODRIGUEZ OCAÑA, 1996; KERRIDGE, 1978; ONDERSTEIJN et al., 2003). Longe de se encontrar um padrão que englobe todas as realidades estudadas, o que se observa é uma complexa diversidade de motivações, valores e crenças aliada aos diferentes ambientes e espaços sociais nas quais os agricultores estão inseridos.

$\mathrm{Na}$ busca de compreender mais profundamende um destes espaços

ParaOnde!?, Porto Alegre, v.13, n.1, p.152-166, 2020.http://seer.ufrgs.br/paraonde 
sociais este estudo se propoe a investigar os regimes orientadores de agricultores da região de Porto Alegre/RS. Embora seja reconhecida a relevância da agricultura neste município, os produtores locais têm enfrentado inúmeras dificuldades advindas principalmente da expansão urbana e aumento da pressão do mercado imobiliário. Tal processo desencadeou grandes transformações no território da cidade, principalmente em suas porções sul, extremo sul e arredores, em municípios adjacentes. Essa região, de características predominantemente rurais e com um longo histórico de atividade agrícola atualmente é alvo de disputa entre a especulação imobiliária e agricultores que tentam se manter na atividade, o que compromete o futuro da agricultura e das famílias produtoras, sendo essas empurradas cada vez mais para longe dos centros urbanos (WANDSCHEER \& MEDEIROS, 2015; KOZENIESKI \& MEDEIROS, 2018).

Neste contexto encontram-se os agricultores de base ecológica pertencentes a Associação dos Produtores da Rede Agroecológica Metropolitana (RAMA). Fundada no final de 2011, a RAMA é o resultado da organização de agricultores de Porto Alegre e Viamão que necessitavam de certificação frente à legislação de orgânicos, em conjunto com consumidores, técnicos da EMATER/RS-ASCAR, prefeituras municipais destes dois municípios, Ministério da Agricultura, Pecuária e Abastecimento (MAPA), Faculdade de Agronomia da Universidade Federal do Rio Grande do Sul (UFRGS) e da Secretaria da Agricultura, Pecuária, Pesca e agronegócio do Estado do Rio Grande do Sul, atual Secretaria da Agricultura, Pecuária e Desenvolvimento Rural (RAMOS et al., 2013).

Suas origens remontam ao final da década de 1970 e inicio dos anos 1980 com a união de consumidores que buscavam alimentos mais saudáveis e ecológicos e agricultores familiares tradicionais descontentes com padrão convencional de produção e mercatilização, os quais iniciaram a formação de diversas cooperativas e associações. Após este longo processo de formação, hoje a RAMA atua como Organismo Participativo de Avaliação de Conformidade Orgânica (OPAC), o qual, é responsável pela certificação orgânica de aproximadamente 45 agricultores familiares oriundos da região metropolitana de Porto Alegre (Figura 1). Além de exercer o papel de certificadora participativa, a RAMA também presta assessoria técnica, promove atividades de extensão rural e auxilia na comercialização e distribuição dos produtos de seus associados.

ParaOnde!?, Porto Alegre, v.13, n.1, p.152-166, 2020.http://seer.ufrgs.br/paraonde 
Figura 1 - Vista de reunião da Associação dos Produtores da Rede Agroecológica Metropolitana (RAMA) realizada em Porto Alegre/RS

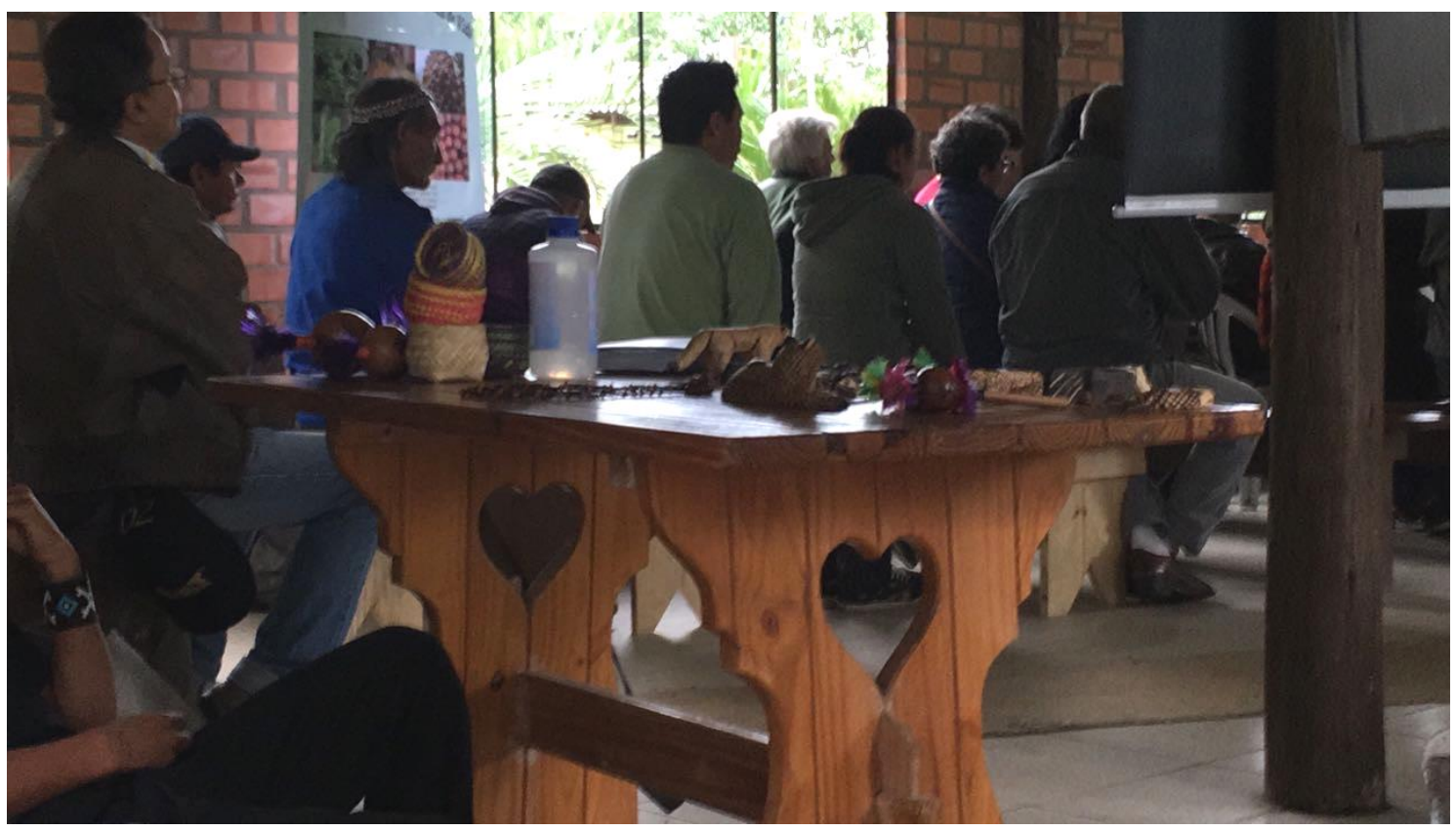

Foto: Autor.

A partir do exposto, fica evidente a necessidade de se compreender os valores e objetivos orientadores do comportamento e das ações dos agricultores locais de forma a entender a lógica por trás da gestão e da organização de suas unidades produtivas antagonistas ao modelo hegemômico de planejamento e ocupação deste território. Mais ainda, tal entendimento busca auxiliar no planejamento de ações de órgãos de assistência técnica e extensão rural (ATER) e políticas públicas que ampliem as capacidades destes agricultores e promovam um desenvolvimento mais inclusivo e justo para a região.

Dessa forma, o presente estudo tem por objetivo aprofundar a compreensão das motivações, comportamento e ações de agricultores pertencentes a Associação dos Produtores da Rede Agroecológica Metropolitana (RAMA), sediada em Porto Alegre, segundo os regimes de orientação de valores e crenças propostos por Gasson (1973).

\section{Procedimentos metodológicos}

O município de Porto Alegre, localizado às margens do rio Guaíba no estado do Rio Grande do Sul, possui uma população estimada de 1.479.101 habitantes e um território de aproximadamente $495 \mathrm{~km}^{2}$ (IBGE, 2017) (Figura 2). Sua área rural, localizada na região sul e extremo sul do município abrange $120 \mathrm{~km}^{2}$, ou seja, $24 \%$ de seu território, sendo considerada a segunda maior em uma capital no Brasil (SHIFINO, 2015).

Figura 2 - Mapa de localização do município de Porto Alegre/RS

ParaOnde!?, Porto Alegre, v.13, n.1, p.152-166, 2020.http://seer.ufrgs.br/paraonde 


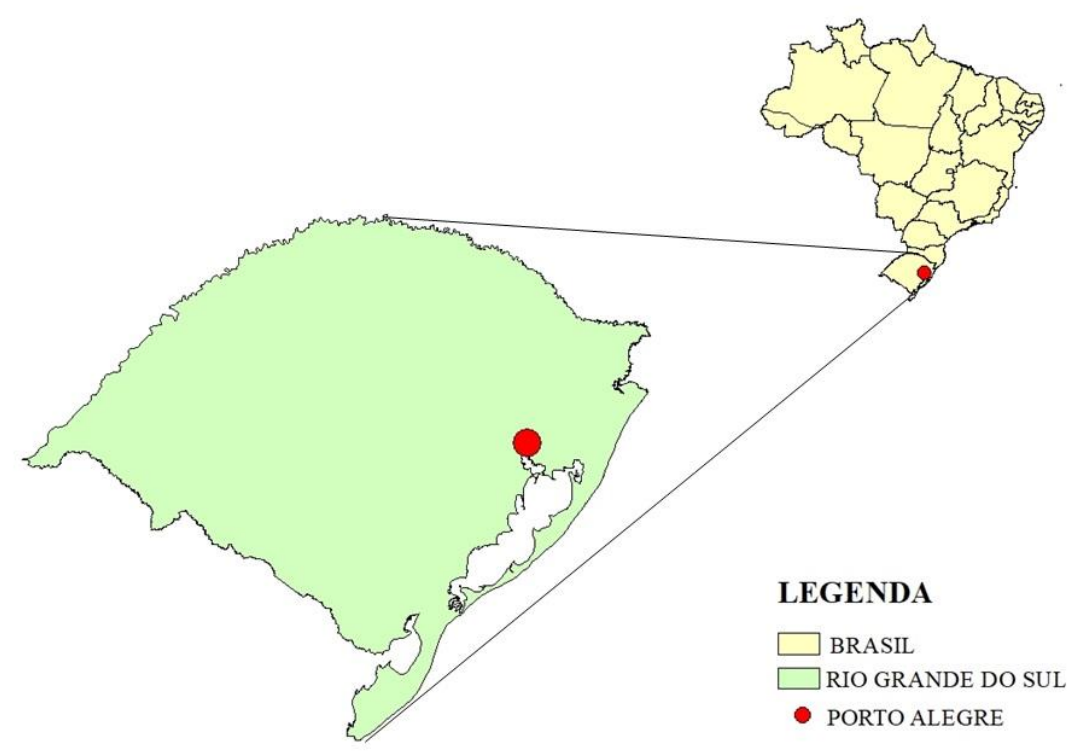

Fonte: Elaboração própria.

No tocante à agricultura, destaca-se que a mesma sempre esteve presente em seu território, ainda que ao longo de sua história tenha sido observada a diminuição dos espaços potenciais para a atividade devido a expansão da cidade. (KOZENIESKI \& MEDEIROS, 2018). Embora possa se identificar algumas conquistas em prol da atividade agrícola no município nos últimos anos, como por exemplo, a reinstauração de sua zona rural pela Lei Complementar no 775/2015, anteriormente extinta pelo seu Plano Diretor de Desenvolvimento Urbano Ambiental (PDDUA) no ano de 1999, a descontinuidade e a não efetivação de muitas iniciativas devido à alternância de governos geram instabilidade e insegurança entre os agricultores portoalegrenses (STEFFENS, 2018).

As propriedades em sua maioria apresentam área inferior a 5 módulos fiscais (25ha), nas quais é produzida uma ampla variedade de produtos hortifrutigranjeiros (KOZENIESKI, 2010). De acordo com Schifino (2015), são 720 estabelecimentos, abrigando 1.263 produtores cadastrados no Sindicato Rural de Porto Alegre, o que demonstram um número expressivo de agricultores na região. No entanto, cabe destacar que observa-se no município uma grande divergência entre dados vindos do Censo Agropecuário, do INCRA e da EMATER/RS acerca de sua estrutura fundiária, não sendo possível identificar de modo preciso o número total de estabelecimentos agropecuários e suas respectivas áreas (KOZENIESKI, 2010).

Observam-se no município duas grandes situações. A primeira caracterizada por uma agricultura empresarial dedicada, sobretudo, a produção de arroz, animais e rações e constituída por poucos estabelecimentos com grandes produções. A segunda representada por agricultores familiares é caracterizada predominantemente por pequenas propriedades e constituem a maior parte dos estabelecimentos rurais do município. Esses possuem uma grande diversidade de formas de produção e de comercialização, podendo ser

ParaOnde!?, Porto Alegre, v.13, n.1, p.152-166, 2020.http://seer.ufrgs.br/paraonde 
diferenciadas entre produção convencional, com produtos comercializados principalmente na CEASA/RS, e produção de base ecológica, na qual a comercialização se dá essencialmente através de feiras ecológicas da cidade (KOZENIESKI \& MEDEIROS, 2018) (Figura 3).

Figura 3 - Vista de área de produção de agricultor de base ecológica pertencente à RAMA, localizada em Porto Alegre/RS

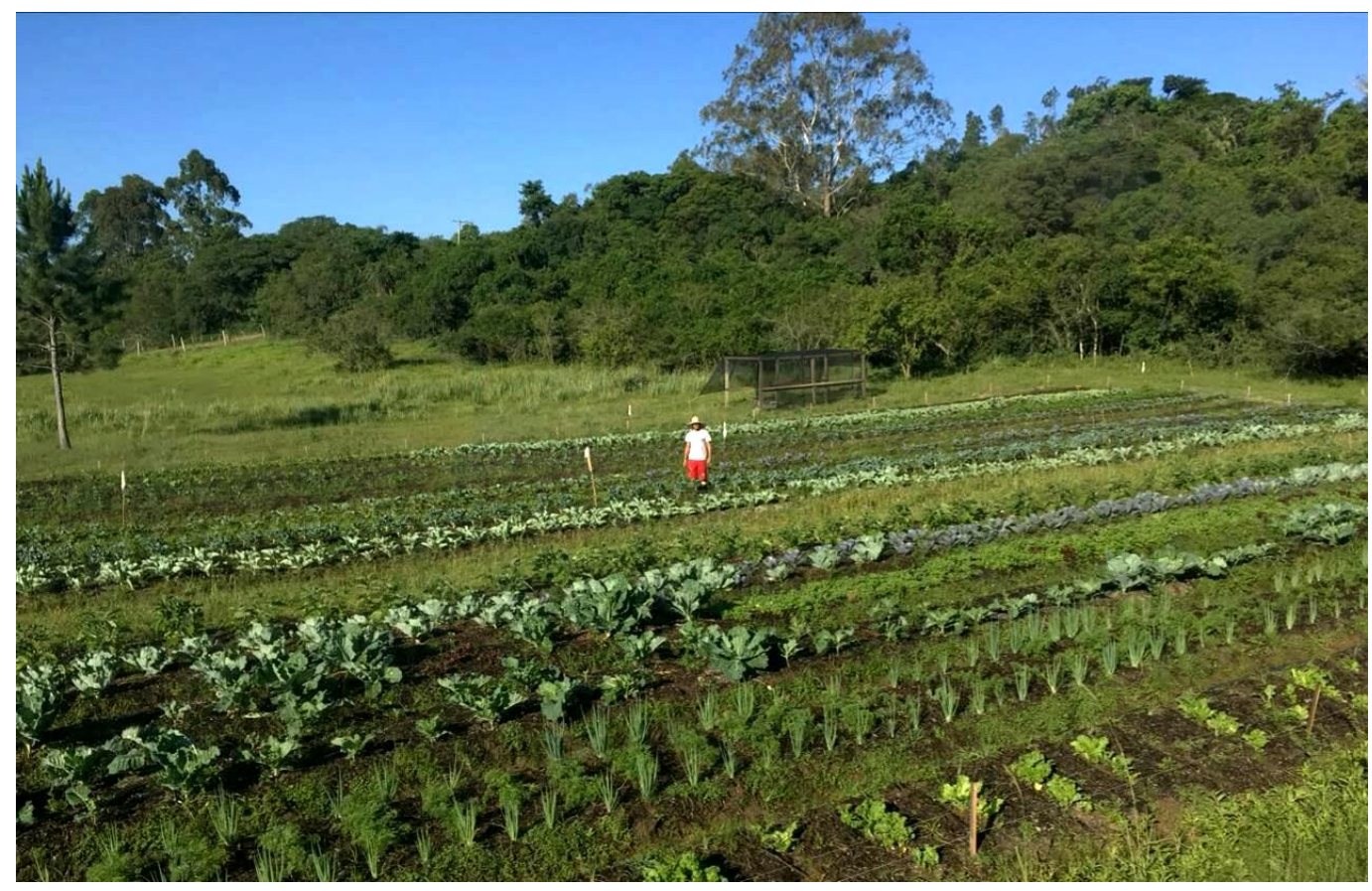

Fonte: Autor

O presente estudo optou por trabalhar com esta última categoria pelo fato da mesma fomentar práticas que trazem consigo benefícios ambientais e sociais para a cidade, além de proporcionar melhor qualidade de vida para os agriculores e garantir o abastecimento de um mercado consumidor em ampla expansão (mercado de orgânicos). Dessa forma, o objeto de estudo contempla os(as) agricultores(as) de base ecológica de Porto Alegre e região, pertencentes à RAMA.

Para responder aos objetivos elucidados, foram realizadas entrevistas semiestruturadas junto a 15 agricultores certificados pela RAMA (14 de Porto Alegre e um de Viamão) durante o mês de junho de 2018, sendo os mesmos selecionados de maneira não-aleatória. O conteúdo abordado envolveu questões acerca do agricultor(a), sua família, sistema produtivo e suas percepções e motivações em relação a prática agrícola e modo de vida, de forma que se permitisse analisar tanto variáveis socioeconômicas e técnicoprodutivas, como também de ordem psicológica e comportamental.

Os valores e motivações influentes no comportamento e ação dos agricultores, conforme os regimes orientadores propostos por Gasson (1973), foram acessados por meio de questões fechadas utilizando-se a escala Likert. Dessa maneira, os entrevistados indicaram o grau de concordância/discordância em relação à uma série de afirmações acerca de suas motivações e objetivos perante a prática agrícola e modo de vida a partir

ParaOnde!?, Porto Alegre, v.13, n.1, p.152-166, 2020.http://seer.ufrgs.br/paraonde 
de cinco categorias de resposta, sendo elas: 1- muito baixa importância; 2 baixa importância; 3 - média importância; 4 - alta importância, e; 5 - muito alta importância.

Por fim, para a análise dos dados levantados foram realizadas estatísticas descritivas, tais como média, desvios-padrão e coeficiente de variação $(\mathrm{CV})$, sendo os resultados apresentados por meio de gráficos e tabelas.

\section{Resultados e discussão}

Por meio das informações obtidas pôde-se traçar o perfil dos agricultores pertencentes à RAMA. A média de idade dos agricultores entrevistados é de 49,8 anos, estando os mesmos em uma faixa etária de 28 a 66 anos, o que indica uma grande amplitude onde podemos encontrar tanto agricultores bem jovens quanto de idade mais avançada. Quase metade das famílias apresentam entre 1 e 2 pessoas (47\%), ou seja, formada apenas por casais. No entanto, podem ser encontradas também familías maiores com mais de 6 integrantes (7\%) (Tabela 1).

Tabela 1 - Composição familiar dos agricultores de base ecológica da RAMA

\begin{tabular}{ccc}
\hline FAMILIAS & $\mathbf{N}^{0}$ & $\%$ \\
\hline $1-2$ pessoas & 7 & $47 \%$ \\
$2-4$ pessoas & 4 & $27 \%$ \\
$4-6$ pessoas & 3 & $20 \%$ \\
Mais de 6 & 1 & $7 \%$ \\
Total & 15 & $100 \%$ \\
\hline
\end{tabular}

Fonte: Elaboração própria.

As propriedades rurais são de modo geral pequenas, com área total média de 6,4ha e áreas produtivas de 2,5ha. No entanto, podem ser observadas também propriedades de maior, com quase $1 / 3$ do total apresentando mais de 10ha (4) (Tabela 2).

\section{Tabela 2 - Tamanho das propriedades e respectivas áreas de produção dos agricultores de base ecológica da RAMA}

\begin{tabular}{cccccc}
\hline \multirow{2}{*}{ TAMANHO } & \multicolumn{2}{c}{ AREA TOTAL } & \multicolumn{2}{c}{ AंREA ÚTIL } \\
& $\mathbf{N}^{\mathbf{0}}$ & $\%$ & $\mathbf{N}^{0}$ & $\%$ \\
\hline $0-2 \mathrm{ha}$ & 7 & $47 \%$ & 11 & $73 \%$ \\
$2-5$ ha & 3 & $20 \%$ & 2 & $13 \%$ \\
$5-10$ ha & 1 & $7 \%$ & 2 & $13 \%$ \\
Maior que 10 ha & 4 & $27 \%$ & 0 & $0 \%$ \\
Total & 15 & $100 \%$ & 15 & $100 \%$ \\
\hline
\end{tabular}

Fonte: Elaboração própria.

ParaOnde!?, Porto Alegre, v.13, n.1, p.152-166, 2020.http://seer.ufrgs.br/paraonde 
Na tabela 3 abaixo são apresentados os resultados obtidos pela análise de 20 variáveis propostas por Gasson (1973) acerca dos valores e objetivos atuantes sobre o comportamento e ação dos agricultores de base ecológica da RAMA. As afirmações com médias elevadas e maior consenso, ou seja, menores desvios padrões e CVs, entre os agricultores foram: satisfação com o trabalho ao ar livre, poder ser criativo no trabalho, satisfação em trabalhar em sua propriedade, independência nas decisões e alcançar os objetivos que se deseja. Embora as afirmações bom relacionamento com os trabalhadores e exercer habilidades e aptidões especiais tenham obtido a mesma média que alcançar os objetivos que se deseja, estas apresentaram maiores desvios padrão e CVs o que demonstra menor homogeneidade de resposta entre os agricultores.

Tabela 3 - Regimes orientadores do comportamento e ação dos agricultores de base ecológica da RAMA, de acordo com Gasson (1973). ${ }^{*} \mathrm{CV}$ - Coeficiente de variação

\begin{tabular}{|c|c|c|c|c|c|}
\hline VALORES E MOTIVAÇÖES & $\begin{array}{l}\text { REGIMES DE } \\
\text { ORIENTAÇÃO }\end{array}$ & MÉDIA & $\begin{array}{l}\text { DESVIO } \\
\text { PADRÄO }\end{array}$ & $\begin{array}{l}\text { CV } \\
(\%)\end{array}$ & $\begin{array}{l}\text { MĖDIA DA } \\
\text { ORIENTAÇÃO }\end{array}$ \\
\hline $\begin{array}{l}\text { Satisfação com o trabalho ao ar } \\
\text { livre }\end{array}$ & INTRINSECO & 4,53 & 0,64 & 0,14 & \multirow{4}{*}{4,35} \\
\hline Independência nas decisões & INTRINSECO & 4,40 & 0,74 & 0,17 & \\
\hline $\begin{array}{l}\text { Aceitar e controlar situações de } \\
\text { risco }\end{array}$ & INTRINSECO & 4,27 & 0,80 & 0,19 & \\
\hline $\begin{array}{l}\text { Valorizar o trabalho duro da lida no } \\
\text { campo }\end{array}$ & INTRINSECO & 4,20 & 0,94 & 0,22 & \\
\hline Poder ser criativo no trabalho & EXPRESSIVA & 4,53 & 0,64 & 0,14 & \multirow{6}{*}{4,30} \\
\hline $\begin{array}{l}\text { Satisfação em trabalhar em sua } \\
\text { propriedade }\end{array}$ & EXPRESSIVA & 4,47 & 0,74 & 0,17 & \\
\hline Alcançar os objetivos que se deseja & EXPRESSIVA & 4,33 & 0,62 & 0,14 & \\
\hline $\begin{array}{l}\text { Exercer habilidades e aptidões } \\
\text { especiais }\end{array}$ & EXPRESSIVA & 4,33 & 0,82 & 0,19 & \\
\hline Satisfação em sentir-se proprietário & EXPRESSIVA & 4,27 & 0,96 & 0,23 & \\
\hline $\begin{array}{l}\text { Flexibilidade em termos de } \\
\text { calendário e horários }\end{array}$ & EXPRESSIVA & 3,87 & 1,25 & 0,32 & \\
\hline $\begin{array}{l}\text { Obter um bom rendimento } \\
\text { (satisfatório) para família }\end{array}$ & INSTRUMENTAL & 4,13 & 0,83 & 0,20 & \multirow{5}{*}{4,03} \\
\hline Condições agradáveis de trabalho & INSTRUMENTAL & 4,07 & 0,80 & 0,20 & \\
\hline Garantir renda para o futuro & INSTRUMENTAL & 4,07 & 0,88 & 0,22 & \\
\hline Maximizar lucro da produção & INSTRUMENTAL & 4,07 & 1,03 & 0,25 & \\
\hline Expandir os negócios & INSTRUMENTAL & 3,80 & 1,15 & 0,30 & \\
\hline $\begin{array}{l}\text { Bom relacionamento com os } \\
\text { trabalhadores }\end{array}$ & SOCIAL & 4,33 & 0,82 & 0,19 & \multirow{5}{*}{3,63} \\
\hline Relação com a comunidade rural & SOCIAL & 4,20 & 0,94 & 0,22 & \\
\hline Trabalho em familia & SOCIAL & 3,80 & 0,68 & 0,18 & \\
\hline Prestígio social por ser agricultor & SOCIAL & 3,33 & 1,29 & 0,39 & \\
\hline Continuar a tradição da família & SOCIAL & 2,47 & 1,25 & 0,51 & \\
\hline
\end{tabular}

Fonte: Elaboração própria.

De acordo com os regimes orientadores de Gasson (1973), o comportamento destes agricultores é norteado por valores e objetivos de ordem intrínseca $(4,35)$ e expressiva $(4,30)$, relacionados principalmente à valorização da agricultura como uma atividade em si mesma, na apreciação do

ParaOnde!?, Porto Alegre, v.13, n.1, p.152-166, 2020.http://seer.ufrgs.br/paraonde 
trabalho no campo e na realização e satisfação pessoal através da prática agrícola. Esta satisfação também pode ser demonstrada pela Figura 4, na qual se observa que mais de $93 \%$ dos entrevistados e suas famílias encontram-se razoavelmente a muito satisfeitos em relação à atividade.

\section{Figura 4 - Grau de satisfação do agricultor(a) e de sua família em relação} a atividade agrícola
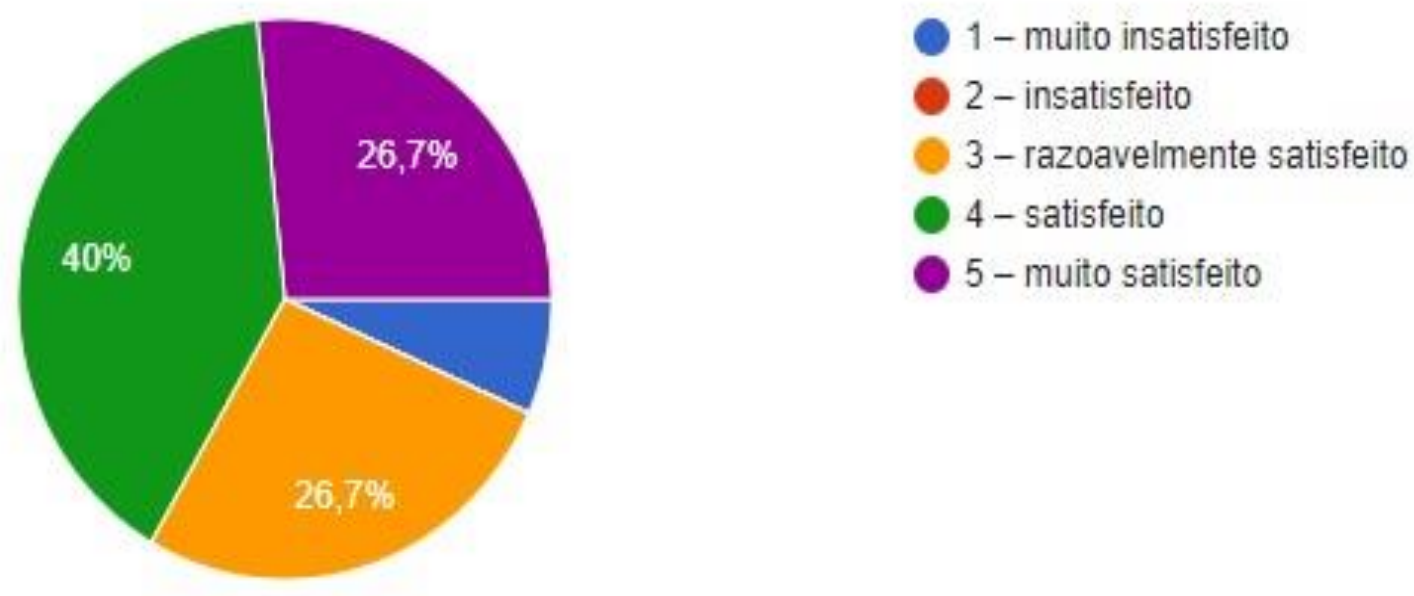

Fonte: Elaboração própria.

Acerca da relação entre tamanho da propriedade e os regimes orientadores, Gasson (1973), na Inglaterra, e Kerridge (1978), na Austrália, observaram que agricultores com menores estabelecimentos priorizavam orientações de caráter instrínseco, ao passo que para médios e grandes proprietários sobressaíram-se regimes instrumentais e sociais. O mesmo esta relacionado aos agricultores de base ecológica da RAMA, os quais na sua grande maioria são considerados pequenos produtores. Diferentemente, nos resultados obtidos por Wives \& Machado (2014) os agricultores com menores áreas tinham uma forte associação ao regime de orientação instrumental.

A respeito do nível de instrução, Kerridge (1978) encontrou relação positiva entre valores intrínsecos e educação, ou seja, quanto maior a escolaridade e acesso a fontes de informação, mais os agricultores valorizavam a agricultura pela independência nas decisões e lida no campo, assim como pela satisfação de se trabalhar ao ar livre. Para o autor, agricultores com maiores níveis de escolaridade teriam mais oportunidades e opções de escolha de trabalho e optariam pela agricultura mais conscientemente. Este cenário também foi observado por Ondersteijn et al. (2003), todavia, não significativo estatisticamente.

\section{Tabela 4 - Nível de escolaridade dos agricultores de base ecológica da RAMA.}

ParaOnde!?, Porto Alegre, v.13, n.1, p.152-166, 2020.http://seer.ufrgs.br/paraonde 


\begin{tabular}{ccc}
\hline ESCOLARIDADE & $\mathbf{N}^{0}$ DE ENTREVISTADOS & $\%$ \\
\hline Ensino fundamental incompleto & 1 & $7 \%$ \\
Ensino fundamental completo & 0 & $0 \%$ \\
Ensino médio incompleto & 0 & $0 \%$ \\
Ensino médio completo & 0 & $0 \%$ \\
Ensino superior incompleto & 4 & $27 \%$ \\
Ensino superior completo & 9 & $60 \%$ \\
Pós-graduação & 1 & $7 \%$ \\
Total & 15 & $100 \%$ \\
\hline
\end{tabular}

Fonte: Elaboração própria.

Esta mesma relação pode explicar os regimes orientadores predominantes no presente trabalho. Entre os agricultores entrevistados observa-se que $67 \%$ deles possuem pelo menos ensino superior completo (Tabela 4). Além disso, os mesmos possuem elevado acesso a informações por apresentarem níveis médio a alto em relação ao hábito de leitura em geral e específica em agricultura agroecológica (tabela 5)

Tabela 5 - Hábito de leitura em geral e específica acerca de agricultura agroecológica dos agricultores de base ecológica da RAMA.

\begin{tabular}{ccc}
\hline ESCOLARIDADE & $\mathbf{N}^{0}$ DE ENTREVISTADOS & $\%$ \\
\hline Ensino fundamental incompleto & 1 & $7 \%$ \\
Ensino fundamental completo & 0 & $0 \%$ \\
Ensino médio incompleto & 0 & $0 \%$ \\
Ensino médio completo & 0 & $0 \%$ \\
Ensino superior incompleto & 4 & $27 \%$ \\
Ensino superior completo & 9 & $60 \%$ \\
Pós-graduação & 1 & $7 \%$ \\
Total & 15 & $100 \%$ \\
\hline
\end{tabular}

Fonte: Elaboração própria.

Motivações de orientação instrumental relacionadas à renda e maximização do lucro encontraram-se em um nível intermediário nas respostas dos nossos entrevistados, com média 4,03, o que corrobora com a afirmação de que a maximização dos lucros advogada pela economia neoclássica não é suficiente para predizer o comportamento dos agricultores no campo. O mesmo resultado é encontrado em outros trabalhos realizados no estado do Rio Grande do Sul, como o de Andreatta (2009), em seu estudo acerca do perfil dos pecuaristas e organização de seus estabelecimentos, e o de Wives \& Machado (2014), sobre fatores influentes na tomada de decisão de produtores de banana no litoral norte do estado. Todavia, cabe mencionar o trabalho de Andrade (2010), que em seu estudo constatou que as motivações no processo de tomada de decisão de produtores rurais de Sant'ana do Livramento/RS e que se dedicam a algum tipo de agricultura é orientada em primeiro lugar por regimes de ordem isntrumental (35,4\% dos entrevistados), seguido por valores sociais (25\% dos entrevistados) e expressivos $(22,7 \%)$.

Para o presente trabalho este resultado pode estar relacionado com o fato de que quase metade dos agricultores amostrados (47\%) exerce outra atividade profissional e/ou possuem alguma outra renda não-agrícola

ParaOnde!?, Porto Alegre, v.13, n.1, p.152-166, 2020.http://seer.ufrgs.br/paraonde 
(aposentadoria/pensão). Todavia, outros fatores também podem ter influenciando em tal resultado. Por pertencerem à RAMA estes agricultores recebem auxílio na veiculação de suas mercadorias em feiras orgânicas do município e no acesso a outros canais de comercialização como, por exemplo, - Plano Nacional de Alimentação Escolar (PNAE) e o Programa de Aquisição de Alimentos (PAA) do governo federal. Além disso, pelo fato dos mesmos encontrarem-se muito próximos a grandes mercados consumidores, como o da cidade de Porto Alegre, pode fazer com que suas motivações sejam outras por terem retornos financeiros satisfatórios garantidos.

Por fim, motivações de caráter social são consideradas de menos importância. O fato de que metade dos entrevistados têm ou teve pais agricultores, sendo que $82 \%$ desses vieram de outras regiões (Figura 5), pode estar relacionado com menores motivações relacionadas à tradição familiar e pertencimento ao território e comunidade local.

\section{Figura 5 - Resultados obtidos acerca das seguintes perguntas direcionadas aos agricultores da RAMA: a) Seus pais eram agricultores?b) Se sim, na mesma região? (Azul - Sim, Vermelho - Não)}

a)

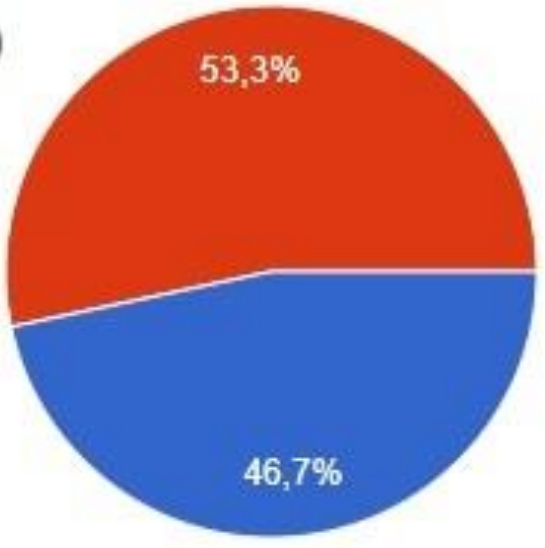

b)

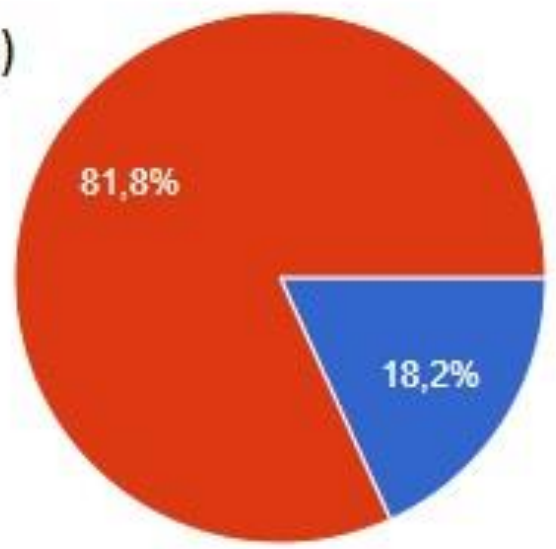

Fonte: Elaboração própria.

Ademais, por se tratarem em sua maioria de famílias pequenas, $47 \%$ com 1 a 2 membros e $27 \%$ com 2 a 4 membros, aspectos como exercer 0 trabalho em família e manter bom relacionamento com os trabalhadores podem ter menos influência. Por fim, Ondersteijn et al. (2003) observaram que agricultores com maiores níveis de instrução, se preocupam menos com 0 prestígio social de sua atividade perante a comunidade em geral, o que também pode estar relacionado com o presente trabalho.

Valores de caráter social apresentaram maior relevância em estudos como de Wives \& Machado (2014), onde a bananacultura no litoral norte do RS é considerada uma atividade de grande tradição familiar, constituindo-se como um reflexo da história e da cultura da região. O mesmo vale para os estudos de SENAR/FARSUL/SEBRAE (2005) e Cezar (1999), os quais observaram o predomínio de valores relacionados à tradição e cultura entre bovinocultores do

ParaOnde!?, Porto Alegre, v.13, n.1, p.152-166, 2020.http://seer.ufrgs.br/paraonde 
RS e do Pantanal, ambas atividades de longa data nas duas regiões em questão.

Em suma, embora possa haver variação na hierarquização ou apresentar maior complexidade na interação das motivações de agricultores $n$ sua tomada de decisao, comportamento e ação, são muitos trabalhos que corroboram com os resultados alcançados para os agricultores de base ecológica da RAMA, o que reafirma o pressuposto de Gasson (1973) que o fator econômico não explica na sua grande maioria o comportamento dos agricultores em suas propriedades. Esta constatação demonstra a necessidade de se considerar aspectos internos relacionados aos agricultores, sua família e situação socioeconômica no fomento de políticas públicas mais efetivas e específicas, assim como na elaboração de projetos de desenvolvimento e ações de órgãos governamentais de assistência técnica e extensão rural (ATER).

\section{Considerações finais}

No presente estudo foram analisados os fatores internos relacionados às características socioeconômicas e comportamentais dos agricultores de base ecológica de Porto Alegre e arredores pertencentes à RAMA. Quando indagados a respeito de valores e objetivos que exercem influência em seu comportamento e ação na gestão e organização de seus estabelecimentos, os mesmos demonstraram maiores motivações de caráter intrínseco e expressivo, em detrimento de aspectos econômicos e culturais. Por se tratarem de pequenos produtores rurais com elevado nível de escolaridade e amplo acesso a informação, os mesmos vêem na agricultura a busca para uma maior qualidade de vida e satisfação pessoal, não vislumbrando obter grandes retornos financeiros ou perpetuar uma tradição familiar. Tal fato reforça a necessidade de se formular políticas públicas e ações governamentais que levem em consideração os motivos que fazem com que estes agricultores persistam em suas atividades, visto que os valores e objetivos que os mesmos carregam consigo contribuem de maneira significativa nas suas ações e comportamento, ao mesmo tempo que moldam o contexto no qual estão inseridos.

\section{Referências}

ANDREATTA, T. Bovinocultura de corte no Rio Grande do Sul: um estudo a partir do perfil dos pecuaristas e organização dos estabelecimentos agrícolas. 2009. 226f. Tese (Doutorado em Desenvolvimento Rural) - Faculdade de Ciências Econômicas, Universidade Federal do Rio Grande do Sul, Porto Alegre, 2009. Disponível em: https://www.lume.ufrgs.br/handle/10183/16416. Acesso em 11/12/2019.

BURTON, R. J. F. Reconceptualising the "Behavioral Approach" in Agricultural Studies: A Socio-Psychological Perspective. Journal of Rural Studies, New York, v. 20, p. 359-371. 2004.

CEZAR, I. M. A Participatory Knowledge Information System for Beef

ParaOnde!?, Porto Alegre, v.13, n.1, p.152-166, 2020.http://seer.ufrgs.br/paraonde 
Farmers: A Case Applied to the State of Mato Grosso do Sul. 1999. 324f. Thesis (Socioeconomy Doctorate), Institute of Ecology and Resource Management, University of Edinburgh, Edinburgh, 1999. Disponível em: https://era.ed.ac.uk/handle/1842/27771?show=full. Acesso em 15/11/2019.

GASSON, R. Goals and values of farmers. Journal of Agricultural Economics, Ashford, v. 24, p. 521-537, 1973.

INSTITUTO BRASILEIRO DE GEOGRAFIA E ESTATÍSTICA (IBGE). Cidades: Porto Alegre. Disponível em: http://cidades.ibge.gov.br/xtras/perfil.php?lang=\&codmun=431490\&search=riogrande-do-sul|porto-alegre|infograficos:-informacoes-completas. Acesso em: 02/09/2019.

KERRIDGE, K. W. Value orientations and farmer behaviour: an exploratory study. Quarterly Review of Agricultural Economics, Caberra, v.31 n.1, p. 61-72. 1978.

KOZENIESKI, É. M., MEDEIROS, R. M. V. O rural agrícola na metrópole: o caso de Porto Alegre/RS. Revista franco-brasileira de geografia, São Paulo, v. $35, \quad$ p. $1-20.2018 .2$ Disponível em: https://journals.openedition.org/confins/13167. Acesso em: 15/9/2019.

KOZENIESKI, É. O Rural agrícola na metrópole: o caso de Porto Alegre/RS. 2010. 140f. Dissertação (Mestrado em Geografia) - Instituto de Geociências, Universidade Federal do Rio Grande do Sul, Porto Alegre, 2010. Disponível em: https://lume.ufrgs.br/handle/10183/25549. Acesso em: 22/10/2019.

MCGREGOR, M.J.; WILLOCK, J.; DENT.J.B.; DEARY, I.; SUTHERLAND, A.; GIBSON, G.; MORGAN, O. AND GRIEVE, B. Links between psychological factors and farmer decision making. Farm Management, v. 9 n. 5, p. 228-239. 1996.

Disponível

em: https://www.researchgate.net/publication/255687361_Links_Between_Psycholo gical_Factors_and_Farmer_Decision_Making. Acesso em 24/11/2019.

ONDERSTEIJN, C. J. M.; GIESEN, G. W. J.; HUIRNE, R. B. M. Identification of farmer characteristics and farm strategies explaining changes in environmental management and environmental and economic performance of dairy farms. Agricultural Systems, Holanda, v. 78, n. 1, p. 31-55. 2003. Disponível em: https://www.sciencedirect.com/science/article/pii/S0308521X03000313. Acesso em: 29/08/2019.

PREFEITURA MUNICIPAL DE PORTO ALEGRE: SCHIFINO, A. Projeto que prevê a volta da Zona Rural é debatido no Legislativo. Disponível em: http://www2.portoalegre.rs.gov.br/portal_pmpa_empreendedor/default.php?p_n oticia $=177855 \& P R O J E T O+Q U E+P R E V E+A+V O L T A+D A+Z O N A+R U R A L+E+D$ EBATIDO+NO+LEGISLATIVO. Acesso em: 02/09/2019.

RAMOS, L. P. V.; FIDLER, S. T.; FRÜHAUF, W; MARQUES, C. L. S.; URIARTT, A. H. Processo de Formação e Consolidação do Organismo Participativo de Avaliação da Conformidade Orgânica (OPAC) da Associação dos Produtores da Rede Agroecológica Metropolitana (RAMA). Cadernos de

ParaOnde!?, Porto Alegre, v.13, n.1, p.152-166, 2020.http://seer.ufrgs.br/paraonde 
Agroecologia, Porto Alegre, v.8, n. 2. 2013. Disponível em: http://revistas.abaagroecologia.org.br/index.php/cad/article/view/14992. Acesso em 15/09/2019.

RODRIGUES, A.; ASSMAS, E. M.; JABLONSKI, B. Psicologia Social, 35 ed. Rio de Janeiro: Editora Vozes, 2015. 584 p.

RODRÍGUEZ OCAÑA, A. Propuesta metodológica para el análisis de la toma de decisiones de los agricultores: aplicación al caso del regadío extensivo cordobés. 1996. 221f. Tese (Doutorado em Economia Agroalimentar) - Escuela Técnica Superior de Ingeniería Agronómica y de Montes, Universidad de Córdoba, Córdoba. 1996.

ROMERO, C.; REHMAN, T. Multiple criteria analysis for agricultural decisions. 2 ed. Amsterdam: Elsevier, 2003. 200p.

SENAR/FARSUL/SEBRAE. Diagnóstico de sistemas de produção de bovinocultura de corte do estado do Rio Grande do Sul. Porto Alegre: IEPE, 2005. 265p.

STEFFENS, C. E. A permanência da atividade agrícola de base ecológica em Porto Alegre. 2018.113f. Dissertação (Mestrado em Desenvolvimento Rural), Faculdade de Ciências Econômicas, Universidade Federal do Rio Grande do Sul, Porto Alegre. 2018.Disponível em: https://lume.ufrgs.br/handle/10183/183300. acesso em 19/10/2019.

WANDSCHEER, E. A. R., MEDEIROS, R. M. V. Agricultura urbana em Porto Alegre: dinâmicas socioeconômicas no espaço local. Geosaberes, Fortaleza, v.6, n.1, p. 298-312. 2015. Disponível em: http://www.geosaberes.ufc.br/geosaberes/article/view/387. Acesso em: 20/10/2019.

WIVES, D. G., MACHADO, J. A. D. Fatores influentes e a tomada de decisão nos sistemas de produção de banana no litoral norte do Rio Grande do Sul. Revista Brasileira de Gestão e Desenvolvimento Regional, Taubaté, v. 10, n. 4 , p. 225-247. 2014. Disponível em: https://www.rbgdr.net/revista/index.php/rbgdr/article/view/1539. Acesso em 05/10/2019. 